\title{
Pharmaceutical and Industrial Traits in Genetically Modified Crops: Co-existence with Conventional Agriculture
}

\author{
GianCarlo Moschini \\ Working Paper 06-WP 429 \\ August 2006 \\ Center for Agricultural and Rural Development \\ lowa State University \\ Ames, lowa 50011-1070 \\ www.card.iastate.edu
}

GianCarlo Moschini is a professor of economics and the Pioneer endowed chair in science and technology policy, Iowa State University.

This manuscript was presented in a principal paper session at the 2006 meetings of the American Agricultural Economics Association, Long Beach, CA, July 23-26, 2006. The comments of Harvey Lapan and Paul Christensen on an earlier draft of the paper are acknowledged. The study was supported in part by the U.S. Department of Agriculture through an NRI grant.

This paper is available online on the CARD Web site: www.card.iastate.edu. Permission is granted to reproduce this information with appropriate attribution to the authors.

Questions or comments about the contents of this paper should be directed to GianCarlo Moschini, 583 Heady Hall, lowa State University, Ames, IA 50011-1070; Ph: (515) 294-5761; Fax: (515) 294-6336; E-mail: moschini@iastate.edu.

The U.S. Department of Agriculture (USDA) prohibits discrimination in all its programs and activities on the basis of race, color, national origin, gender, religion, age, disability, political beliefs, sexual orientation, and marital or family status. (Not all prohibited bases apply to all programs.) Persons with disabilities who require alternative means for communication of program information (Braille, large print, audiotape, etc.) should contact USDA's TARGET Center at (202) 720-2600 (voice and TDD). To file a complaint of discrimination, write USDA, Director, Office of Civil Rights, Room 326-W, Whitten Building, 14th and Independence Avenue, SW, Washington, DC 20250-9410 or call (202) 720-5964 (voice and TDD). USDA is an equal opportunity provider and employer.

Iowa State University does not discriminate on the basis of race, color, age, religion, national origin, sexual orientation, gender identity, sex, marital status, disability, or status as a U.S. veteran. Inquiries can be directed to the Director of Equal Opportunity and Diversity, 3680 Beardshear Hall, (515) 294-7612. 


\begin{abstract}
This paper discusses the implications of using genetically modified crops to biomanufacture pharmaceuticals and industrial compounds from the perspective of their co-existence with conventional agriculture. Such plant-made pharmaceuticals and plantmade industrial products rely on exciting scientific and technological breakthroughs and promise new opportunities for the agricultural sector, but they also entail novel risks. The management of the externalities and of the possible unintended economic effects that arise in this context is critical and poses difficult questions for regulators.
\end{abstract}

Keywords: agriculture, biopharming, co-existence, externalities, genetically modified products, liability, molecular farming, regulation. 


\section{Introduction}

The first ten years of commercial experience with genetically modified (GM) crops must be considered a success, albeit a qualified one. Adoption has been remarkable: first commercially planted in 1996, world GM crop planting reached 220 million acres in 2005 (James 2005). But large-scale adoption has been confined to a few crops grown in only a few countries, and the currently widely grown GM crops are all based on only a handful of socalled input traits that confer herbicide tolerance and/or pest resistance. Furthermore, this technology has been met with hostility by some consumer groups and segments of the public. And, the introduction of GM crops means that supplying non-GM products now requires (relative to the pre-innovation situation) additional costly identity preservation and segregation activities (Bullock and Desquilbet 2002). Thus, the innovation process has, in this context, brought about a new market failure, an externality on the production of conventional (non-GM) products (Lapan and Moschini 2004). Such unintended economic implications of the introduction of GM crops are central to the current debate about the "co-existence" of GM and non-GM agriculture.

The externalities of GM products are also likely to affect the expected introduction of the second generation of GM products (with output traits meant to improve the quality of agricultural products), and may indeed be critical for the third generation of GM products that attempt to biomanufacture pharmaceuticals and industrial compounds in traditional crops. Plant-made pharmaceuticals (PMPs) and plant-made industrial products (PMIPs) promise exciting scientific and technological breakthroughs and new opportunities for the agricultural sector, but also entail novel risks and pose difficult questions for regulators. Some of the questions that arise in this context are the same as with current GM products, including the critical importance of consumer/public aversion and the problem of effective segregation of GM and non-GM products. But PMPs and PMIPs raise enough novel questions to warrant a specific analysis.

\section{Co-existence}

Opposition to GM products has arguably been strongest in the European Union (EU), resulting, inter alia, in a sweeping new regulatory framework that took effect in April 2004. The new EU system - which is meant to foster food safety, protect the environment, and ensure consumers' right to know-is centered on the notions of mandatory labeling and 
traceability of all food and feed produced from GM products (European Union 2004). Perhaps in recognition of the interdependence and externalities of GM crop adoption, noted earlier, the EU is also developing measures aimed at the co-existence of GM and non-GM agriculture:

The issue of co-existence refers to the ability of farmers to provide consumers with a choice between conventional, organic and GM products that comply with European labelling and purity standards. Co-existence is not about environmental or bealth risks because only GM crops that have been authorized as safe for the environment and for human bealth can be cultivated in the EU. ... Co-existence is concerned with the potential economic loss through the admixture of GM and non-GM crops which could lower their value ... (European Union 2003).

Thus, the unintended economic implications of the introduction of GM crops are central to the EU notion of co-existence. In particular, possible health and environmental risk considerations are assumed away by the presumption that they are essentially separable from the possible economic impacts of GM crop adoption (and are dealt with earlier, at the approval stage).

More broadly, the concept of co-existence in agriculture can be defined with reference to the level of adventitious commingling that inevitably besets farm production, given that it takes place in an open and interconnected environment. As such it is not a new issue; it has always been a facet of a diversified agricultural and food system. In this setting, co-existence is not perceived as a problem when crops intended for different markets can be grown and commercialized within the same production and marketing system without compromising the economic value of the products. Thus, co-existence relates to the possibility of growing different crops close enough to each other (e.g., in the same region, or in adjacent farms and/or plots) while the extent of commingling is held at a low enough level. But it is more than that, because adventitious presence may arise from the sharing of infrastructures and other quasi-public inputs throughout the handling, processing and distribution system. The seed industry, for example, has had to deal with commingling issues for a long time, and has developed a number of procedures to meet pre-established purity levels. Whether the coexistence of GM and non-GM crops can be viewed in that way clearly depends on the management of the externality effects in question, that is, on what is an acceptable level of 
adventitious contamination. The EU answer to this question is the $0.9 \%$ threshold established to avoid the need for a GM label of the final product.

\section{Molecular Farming}

Transgenic crops rely on the possibility of introducing foreign DNA into plant cells such that the whole plant can then express new proteins. The first generation of GM crops has targeted the introduction of DNA, often a single gene, which confers an attribute of interest such as herbicide tolerance or insect resistance. This allows the production of essentially the same product (e.g., soybeans or maize) but under a more efficient production system, thereby grounding these products in the long tradition of crop variety enhancements that led to the green revolution (Evenson and Gollin 2003). But genetic transformation techniques can also be used to introduce into plants DNA that codes for the expression of novel proteins that are of direct interest. This allows the host plant to be used in "molecular farming," whereby the intended harvest products are high-value recombinant proteins and organic molecules that can be used in the production of pharmaceutical compounds, as well as recombinant enzymes and polymers with industrial application.

Pharmaceutical applications are of particular interest, given the potential to tap the sizeable and growing market of biotechnology drugs, ${ }^{1}$ leading to the notion of "biopharming." The idea here is that suitably engineered GM plants could be very competitive because alternative production platforms being used and/or researched in the biotechnology industry have well-known drawbacks. For example, microbial fermentation is common but its usefulness is limited by the need for downstream processing because bacteria and yeasts are not capable of certain post-translational modifications (e.g., glycosylation) that are necessary for the biological activity of most complex proteins of biopharmaceutical interest. Recombinant mammalian cell lines, cultured in bioreactors, overcome most of these problems and currently represent the platform of choice for large and complex proteins. But this bio-manufacturing method is very costly. Transgenic plants,

\footnotetext{
1 Insulin, produced by bacteria engineered with recombinant human DNA, was the first biopharmaceutical to be introduced to the market in 1982. As of December 2005, a total of 254 biotechnology drugs have been approved by the U.S. Food and Drug Administration (FDA), with more than 300 new biotech drug products and vaccines currently in clinical trial.
} 
because they possess a eukaryote protein synthesis pathway virtually identical to that of animals, in principle provide an appealing alternative. A suitably transformed GM host plant essentially acts as a bioreactor and can produce recombinant proteins more efficiently than can mammalian cell lines (Ma, Drake, and Christou 2003). ${ }^{2}$

Technical proteins that provided an early proof-of-concept test for the feasibility of PMPs and PMIPs have already resulted in some commercial production (Howard 2005). These products include avidin (a protein usually found in egg whites and useful as a diagnostic reagent), aprotinin (a protease inhibitor found in bovine lungs), and trypsin (a proteolytic enzyme originating in the pancreas, widely used as a reagent). All three can be produced in corn, and aprotinin can also be produced in tobacco. Trypsin produced from corn, developed by Prodigene using bovine DNA, is being marketed by Sigma-Aldrich under the trademark name of TrypZean. Many more products are being developed and are at various stages of the R\&D and production pipeline.

\section{Antibodies}

Monoclonal antibodies (mAbs) are immunoglobulins produced artificially from a single cell clone and thus endowed with great specificity (each recognizes only one antigen). They have use in the diagnosis, prevention, and cure of disease, including cancer detection and therapy. This is one of the most promising segments of the biotechnology industry. To date, the FDA has approved $18 \mathrm{mAbs}$ and more than 150 are in clinical trial. A number of mAbs have been expressed in plants. Tobacco has been used recurrently in research, but other plants used include potatoes, soybeans, rice, and corn (Goldstein and Thomas 2004). In addition to whole plant expression, mAbs are also producible with other plant-based expression systems, such as agrobacterium infiltrated leaves, plant cell cultures and virus-infected plants. Some of these plant-based mAbs are already at advanced levels of clinical trials, and it seems that antibodies may turn out to be the first really successful kind of plant-derived therapeutic proteins (Ma, Drake, and Christou 2003). One critical consideration is that mAbs are often needed in substantial amounts, and thus the demand for mAbs is expected to intensify in the

\footnotetext{
${ }^{2}$ Other platforms are also being used or contemplated, including insect cell cultures, plant cell cultures, and transgenic animals.
} 
near future. The scalability of a viable plant-based production system could thus be extremely valuable.

\section{Vaccines}

Some of the earlier interest in producing vaccines in plants was the vision of obtaining a direct oral-delivery system that represented a low-cost alternative for vaccination. But effective vaccination requires controlled delivery in standardized doses of the active ingredient. Furthermore, triggering the desired immune response from an orally ingested vaccine is difficult, and indeed only one human vaccine (the Sabin polio vaccine) is routinely given in this form. The practicalities of the matter therefore suggest that the hopeful conjecture of an easy oral vaccine may be problematic. Still, production of many subunit vaccines (the purified antigenic determinant that is separated from the virulent organism) is being pursued via plant-based platforms. Four such vaccines are in phase I clinical trial stage: for hepatitis B (expressed in both lettuce and potato), for the Norwalk virus causing diarrhea (expressed in potato), for E. coli heat labile toxin causing diarrhea (expressed in both maize and potato), and for rabies (expressed in viral vector in spinach) (Ma et al. 2005).

In addition to vaccines meant for humans, plant-based vaccines are being developed for use in animal health as well. In fact, edible plant-based vaccines are arguably best suited for this type of application, as an edible product can be conveniently added to animal feed, and even partial protection may be acceptable and economical in that setting. One such vaccine developed by Prodigene is for the transmissible gastroenteritis virus affecting swine, which is expressed by maize that is directly fed to pigs (Howard 2005). A plant-based vaccine to protect poultry from Newcastle disease virus (NDV), developed by Dow, was approved by the USDA-APHIS' Center for Veterinary Biologics in 2006. This is a notable milestone because it is the first-ever plant-based vaccine to win regulatory approval. This vaccine is not expressed in whole plants, however, but is produced by means of GM plant cells cultured in steel fermenters, a production choice that resolves many issues related to containment.

\section{Industrial Products}

Given the ability to engineer new protein expression in plants, the range of potential applications is obviously broader than pharmaceuticals. In fact, a number of PMIPs are 
being pursued, although their development does not appear to be as advanced as for PMPs. Enzymes that can be used in detergents, industrial products or food manufacturing, constitute a fairly large market and are a promising target. One such enzyme developed by Prodigene and expressed in maize is laccase, which acts on lignine and could have applications in paper and textile production. Using genetic engineering to produce enzymes is not new. Chymosin, for example, is an enzyme essential in cheese production (it acts on the milk protein casein causing it to clump) that was traditionally obtained as rennet (rendered from the fourth stomach of milk-fed calves). Recombinant chymosin can now be obtained from GM microorganisms (bacteria and yeasts), and in 1990 it was granted GRAS (generally recognized as safe) status by the FDA. ${ }^{3}$

Other industrial products being engineered into plants include expression of silk proteins from spiders and insects, mammalian-derived structural proteins such as elastin and collagen, and biodegradable plastics that could be an alternative to petroleum-based polymers (Scheller and Conrad 2005). Transgenic crops with specific traits meant to optimize certain properties for bioenergy production are also attracting interest.

\section{U.S. Regulations}

The basic institutional structure for regulating all biotechnology products in the United States is the "Coordinated Framework for Regulation of Biotechnology" established in 1986 (see, e.g., Pew Initiative 2004). This framework involves three federal agencies: the USDA's Animal and Plant Health Inspection Service (APHIS), which regulates the importation, interstate movement, and field testing of GM plants; the FDA, which regulates food and feed additives, human drugs, and medical devices; and the Environmental Protection Agency, which regulates the use of all pesticides, including those expressed in transgenic plants.

Because all GM plants are or might be plant pests, they are considered "regulated articles" by APHIS. Use of such articles outside a contained facility (e.g., in a field test)

\footnotetext{
${ }^{3}$ In the EU chymosin was the first enzyme from a GM organism to be approved for use in food preparation. Interestingly, cheese produced with chymosin is exempted from the mandatory labeling of GM products imposed by the EU, which draws a fine distinction between something produced "with" a GM product (such as cheese, which does not need to be labeled) and something produced "from" a GM product (such as vegetable oil, which needs to be labeled).
} 
requires an authorization that, for all GM plants, used to be obtained through a "notification" procedure. Starting with the 2003 crop year, on the heels of the 2002 Prodigene incident, the introduction of a plant for pharmaceutical or industrial intent is prohibited unless explicitly authorized under a "permit" procedure (USDA/APHIS/BRS 2006). The information required for a permit is more extensive than for a notification, and a permit authorization also entails a number of explicit restrictive conditions. Tight cropspecific measures to ensure containment are envisioned, including isolation distance of test plots (for maize, for example, the distance is one mile, that is eight times the distance that is required for the production of foundation seeds), planting of buffer borders of non-GM crops, and perimeter fallow zones. In addition, the use of dedicated equipment is mandated, there are post-harvest restrictions on the land used, and APHIS also performs a number of inspections during the field test growing season.

A major distinction between plants for PMPs and PMIPs and other GM plants fieldtested under the notification procedure is that the latter can, upon successful experimental releases, petition for non-regulated status. Such a deregulated GM crop can then be freely commercialized with no further oversight by APHIS, and this is in fact the route that has been used for all the major commercial GM crops currently on the market. But no GM plant field-tested under the permit procedure has so far been granted non-regulated status by APHIS.

The role of the FDA arises because the FDA is responsible for regulating human and animal drugs and human biological products. The FDA is particularly concerned that plantmade ingredients meant for therapeutic use meet the same quality standards of safety, purity, potency, and effectiveness as more traditionally made pharmaceuticals. Another role for the FDA in this setting can arise if the GM plant meant for PMPs or PMIPs ends up in the food supply. Should that happen, it seems that the FDA would have to deem the contaminated food to be adulterated, which should trigger recall actions. This effectively establishes a "zero tolerance" level for PMPs and PMIPs in food products.

\section{PMPs, PMIPs, and Co-existence}

It is quite clear that the prospect, and actual advent, of PMPs and PMIPs raises a number of issues that are germane to the debate on the co-existence of GM and non-GM agriculture. One thing that is clear is that here we are dealing with new GM crops that are not intended 
for use in food and feed, unlike current first-generation GM products (or second-generation GM products that pursue quality enhancement of otherwise standard food and feed). Because many of these products are being pursued with host plants that are also used in food and feed production (such as maize and rice), the possibility of adventitious presence of PMP and PMIP traits in the food and feed system becomes a real possibility. The two major avenues by which this could happen are pollen and seed dispersal. Pollen is dispersed mainly by wind and insects. Seeds are dispersed by wind, animals and human activities associated with seed production, harvest, transportation, storage, and processing.

For first-generation GM products co-existence does not bear on health or environmental risk. The reasoning is that the health and environmental safety of GM products is evaluated prior to approval. If approved, then co-existence issues are addressed (e.g., good production practices to minimize adventitious contamination, tolerance levels, assignment of property rights and legal liability rules to induce proper internalization of the externality). But for PMPs and PMIPs this appealing separability does not apply. PMPs and PMIPs may be quite safe, indeed beneficial, for their intended use, but they may also be potentially toxic otherwise (e.g., they may be harmful to humans, animals, and wildlife if ingested). Thus, avoiding the unwanted presence of PMPs and PMIPs in the food and feed system is at present believed to be a necessity, and indeed implicitly mandated by current regulation. That is why containment of PMPs and PMIPs is critical and will require a number of measures that are not necessary with first-generation GM products.

\section{Containment}

A three-pronged attack of the problem is possible. First is spatial separation, as envisioned for example by the isolation requirements of current APHIS permit regulations. More drastically, one could grow PMP and PMIP crops where similar food and feed crops are not grown (e.g., irrigated portions of a desert region). Second is temporal separation, that is, planting PMP and PMIP crops at different times than food and feed crops to minimize the chances of cross-pollination. Third is biological confinement, a strategy emphasized by a recent National Research Council report (NRC 2004). For example, gene flow could be limited by using a male sterile plant host (already a feasible possibility for maize). Limiting the expression of PMP or PMIP traits in chloroplast DNA (which, being part of the 
cytoplasm, are inherited maternally) also reduces the possibility of gene flow. ${ }^{4}$ A more drastic measure of biological confinement concerns the choice of a plant host. Selecting non-food crops for transgenic transformation aimed at PMPs and PMIPs (e.g., tobacco instead of maize) would provide obvious containment advantages.

Another possible containment measure is indoor production. For example, scientists at Arizona State University are working on the expression of vaccine-oriented transformations in tomatoes, a host plant that is well suited for greenhouse production. Note that indoor research and production of PMP and PMIP crops does not have to take place under the APHIS permit regulations discussed earlier (which govern interstate movement, importation, and release in the open environment, as in field tests). Research on GM plants, as well as other genetically engineered organisms, done in contained facilities such as laboratories and greenhouses follow the safety guidelines established by the National Institutes of Health (and such activities, unlike those regulated by APHIS permits, do not need prior approval).

Containment can be affected by the choice of biopharmaceutical production platform. Whole plant expression of PMP and PMIP traits is just one possibility. Cell cultures of transgenic plants (as used in the recently approved vaccine for the NDV in poultry), for example, can be carried out in more traditional bioreactors (similarly to existing bacteria, yeasts of mammalian cell culture expressions) and thus offers considerable containment advantages. Finally, containment provisions are also mandated by the FDA to ensure the purity and safety of the pharmaceutical ingredient that is being produced (and thus not necessarily to prevent adventitious contamination of other products).

\section{Zero Tolerance}

What distinguishes the regulation of GM products meant for PMP and PMIP production, at present, is the implicit zero-tolerance threshold that is envisioned for their presence in nonintended uses such as food and feed. Meeting a zero-tolerance level is difficult and essentially impossible to achieve with probability one. That is a conundrum, but one that is at present upheld under current regulations in the United States as well as abroad. The need for an effective zero tolerance in the food system for transgenic product not approved for food consumption is accepted by the food industry, with some apprehension. The Grocery

\footnotetext{
${ }^{4}$ Chloroplast transformation is of interest in this setting for productivity reasons as well (Fischer et al. 2004).
} 
Manufactures of America and the National Food Processors Association have taken a position against the use of food/feed crops for pharmaceuticals. Because it is widely accepted that $100 \%$ purity is not attainable, ${ }^{5}$ a zero tolerance standard raises the question of what should happen in those (inevitable) events when it is violated. Costly recalls of adulterated food may be necessary, firms may be exposed to consumer and public backlash, and liability issues would inevitably arise.

\section{Liability}

The dawn of biotechnology and the spread of GM crops cultivation create the potential for new liabilities in the agricultural and food system (Smyth et al. 2004). In the United States, persons who believe that their crop or property suffered damage from an activity involving a GM crop (by pollen flow, for example) could bring a tort liability suit under various legal presumptions, including trespass, negligence, or private nuisance (Kershen 2004). Tort claims based on strict liability (i.e., liability without fault) may also be possible, perhaps more so for PMPs and PMIPs than for first-generation GM products. Furthermore, as noted earlier, export market access may be restricted when a GM trait not approved there is found in the U.S. supply (even if in trace levels). In such a case, individuals could bring liability suits based on the concept of public nuisance. ${ }^{6}$

\section{Externalities and the Economics of Co-existence}

Earlier studies documented sizeable efficiency gains from new GM crops (e.g., Moschini, Lapan, and Sobolevsky 2000; Falck-Zepeda, Traxler, and Nelson 2000), but they did not account for the fact that this new technology may not be uniformly accepted by consumers. Once the costs of segregation and identity preservation that are necessary to deliver non-GM products to consumers are considered, the calculus of the economic effects of GM innovations is seriously affected, as discussed and illustrated in Fulton and Giannakas (2004), Sobolevsky, Moschini, and Lapan (2005), and Moschini, Bulut, and Cembalo (2005). The

\footnotetext{
${ }^{5}$ Episodes of accidental GM contamination support this conclusion, including the highprofile StarLink and Prodigene cases (see Taylor and Tick 2003 for a summary).

${ }^{6}$ In the StarLink case farmers claimed economic damages arising because the introduction of GM crops affected export market access and market price for their nontransgenic crop (Kershen 2004).
} 
latter study focuses explicitly on the problem of co-existence, as arising with first-generation GM products, within a multi-market equilibrium model of the EU agricultural sector. The results suggest that the large-scale introduction of GM products in the EU market could reduce overall EU welfare. The magnitude of the economic effects due to GM innovations depends critically on the presence and extent of the costs of segregation, and on the specification of consumer preferences. When all consumers are perfectly indifferent between GM and non-GM products, or when segregation is costless, the model yields the standard result that innovation is welfare-increasing.

How the foregoing results apply to the case of PMPs and PMIPs is, however, an open question (Graff and Moschini 2004; Elbehri 2005). As noted, the essential feature of coexistence is the management of an externality (adventitious contamination). A critical element of such management is the existence of an explicit (and attainable) purity level. For example, in the EU, the maximum allowed GM presence in a non-GM product is $0.9 \%$. But the zero tolerance for PMPs and PMIPs essentially mandates 100\% purity. The expected costs of meeting a given purity level increase exponentially as the standard tightens. Because a non-negligible production of PMP and PMIP crops will necessarily result in commingling incidents with positive probability, one needs to account for the costs of retrieving and disposing of tainted supplies, and the compensation for harm and possibly for the indirect costs (market disruptions, rejection of export shipments, consumer backlash on implicated brands, etc.). The StarLink incident may be instructive: the final cost to Aventis (the seed company that commercialized StarLink) has been estimated to exceed $\$ 1$ billion.

Given the above, some have called for the establishment of non-zero tolerances (e.g., Miller and Longtin 2004). In principle such determination may be done on a case-by-case basis and could reflect familiar science-based principles. That, of course, would require changing the current regulatory framework. To be sure, in 2004 APHIS published a notice of intent to prepare an environmental impact statement evaluating its biotechnology regulations and requesting public comments on a variety of possible changes. These include whether the current one-size-fits-all permit and notification system could be tailored to reflect relative levels of potential risk. It is unclear at this point whether the possibility of deregulating certain pharmaceutical crops will be considered. In an increasingly globalized economy, however, relaxing the current implicit zero tolerance level for PMPs and PMIPs in the U.S. food and feed system may not suffice anyway. As long as there is the possibility for 
shipments of commodities to be rejected by foreign markets, failure to meet the zero tolerance standard could still have sizeable economic impacts.

The costly recalls associated with the StarLink fiasco has many operators concerned about such an eventuality. One solution would be to have the FDA issue a pre-market approval of the product in question as a food additive (even if, in fact, it is not intended for food use). But such a route, which would require establishing acceptable safety levels for consumers, is itself lengthy and costly. It is also doubtful that such a course of action would be acceptable to the food industry, given the potential for a negative consumer reaction, and FDA officials also do not appear interested in this approach (Becker and Vogt 2005).

A critical question in the management of the adventitious contamination externality concerns the market incentive for segregation. For current GM crops that yield a product that is an imperfect (weakly inferior) substitute for the conventional one, the incentive for carrying out the necessary segregation and identity preservation activities falls on the producers of the conventional product, that is, the presumed superior product from the consumers' perspective (Lapan and Moschini 2004; Moschini, Bulut, and Cembalo 2005). For PMPs and PMIPs, on the other hand, the expectation is that the user of the new technology would be responsible for ensuring the adequate containment. This is certainly what is entailed by the current regulations, which also establish a potential legal liability burden on the innovators. This observation has clear implications for the nature of the coexistence that is likely to emerge. Whether plant-based systems can significantly tap the growing biopharmaceutical market will eventually depend on the relative cost of plant-based production compared to alternative platforms. Scientists promoting PMPs have touted the potential efficiency gains of plant-based production. Such hopeful views, however, systematically ignore the cost of internalizing the externalities of potential commingling. Any realistic accounting of the cost of effective containment suggests that open field production of PMPs and PMIPs is a distant possibility at best. In the intermediate run, likelier scenarios may involve more contained production systems, such as the use of plant-cell cultures in traditional closed-vessel fermentation facilities, or the cultivation of transgenic non-food crops in confined greenhouses.

The legal liability under the strict regulations governing PMPs and PMIPs is likely to add substantially to the expected cost of introducing these products. Liability rules are important in a market economy because they provide incentives for responsible choices that 
reduce the external impacts of private activities. But the cost of legal liability suits can be high and thus this may be a costly way to ensure adequate stewardship in the introduction of new biotechnologies. Furthermore, in a world of limited liability where risky actions are taken by small startup companies or individual researchers working in public institutions, as in the case of PMPs and PMIPs, the question remains as to whether the social costs of external effects are properly internalized by the expectation of potential liabilities. The choice of the plant host for PMPs is a good example. Most observers agree on the advantages of avoiding the use of food crops, at least for the time being. But the choice of the plant host to use for PMPs is currently made by early-stage researchers who arguably have little incentive to account for the potential downstream externalities, and for whom the objective of being first (an outcome that carries clear rewards, given the working of the intellectual property rights system) is greatly enhanced by the choice of an established crop.

A distinct issue concerns the expected economic impacts on primary agriculture. Some have touted biopharming as a great opportunity to add significant value to farm production whereas others are skeptical of such a prospect (Wisner 2005). Even if plant-based biopharmaceuticals are successful, ${ }^{7}$ agriculture's direct contribution to this emerging industry may be limited. While PMPs and PMIPs are potentially highly valued, they are likely to require little land to satisfy demand. ${ }^{8}$ Furthermore, to comply with the expected stringent regulation, companies developing these crops are also likely to maintain tight control on the entire production cycle of the products, limiting farmers' direct participation in production. The cost of containment may also induce crop biomanufacturing firms to locate away from traditional areas of agricultural production, possibly outsourcing overseas. Precisely because they have a strong comparative advantage in food and feed production, regions such as the Midwest may be at a competitive disadvantage in growing PMPs and PMIPs. In the final analysis the returns to agriculture from molecular farming will be for use of the land and other services provided in the growing of the crop, a relatively small contribution in the production chain for the PMPs and PMIPs of interest to end users.

\footnotetext{
${ }^{7}$ This is still not a foregone conclusion, if anything because the pharmaceutical industry is showing great caution toward this technology.

${ }^{8}$ This observation should probably be qualified in that PMIPs actually hold the possibility that some high-volume, high-acreage products may emerge, such as GM crops for biomass with suitable properties for biofuel production.
} 


\section{Conclusion}

The trajectory of current plant and biopharmaceutical R\&D suggests that the co-existence of PMPs and PMIPs with traditional agriculture is not only likely, it is inevitable. But the extent and the form of the co-existence that will eventually emerge are bound to be influenced crucially by the management of the possible externalities that arise. A major issue with plantbased biomanufacturing is the potentially large risks that are involved. Although such GM crops are meant to be grown and handled separately, compounds intended for pharmaceutical or industrial use could end up in the food and feed supplies by accident. Direct harm to human health and the environment is deemed unlikely at present (Peterson and Arntzen 2004), but the eventuality of a contamination could be catastrophic in economic terms. Segments of the public and the food industry are particularly concerned and favor strict regulation of these new products (UCS 2004). Arguably, the biotech industry should also be concerned with the risks of PMPs and PMIPs, as an accident in this context would provide powerful ammunition to an already active anti-biotech lobby and could have serious adverse effects on future biotechnology research.

U.S. regulation in this area at present presumes a zero tolerance level for PMPs and PMIPs in the food supply. This strictest of standards requires onerous containment activities and provides an incentive for the technology's developers to maintain effective control on the production of such GM crops in a tight vertically integrated structure. Coupled with the fact that PMPs or PMIPs are likely to require only a small amount of land to satisfy demand in the foreseeable future, this may limit agriculture's direct contribution to the emerging biopharming industry. Options for regulators to consider include whether a strict spatial segregation should be mandated (e.g., do not grow PMP corn in the Corn Belt), or whether the use of food and feed crops to produce PMPs and PMIPs should simply be banned. Conversely, there is the question of whether zero adventitious presence can in fact be achieved in practice; because such a stringent requirement may end up imposing unnecessary costs without increasing safety, it may be desirable to explore ways to allow the adventitious presence of PMPs and PMIPs at low enough levels that risks are minimal. In any event, the stricter containment standards that apply, and will likely continue to apply, to PMPs and PMIPs mean that segregation methods being developed for first-generation GM products may not be particularly valuable in this setting. The co-existence of traditional agriculture 
with crops transformed to express pharmaceutical and industrial traits will therefore take quite a different form than with the first generation of GM products. 


\section{References}

Becker, G.S., and D. Vogt. 2005. "Regulation of Plant-Based Pharmaceuticals." CRS Report for Congress. Order Code RS21418, March 8.

Bullock, D., and M. Desquilbet. 2002. "The Economics of Non-GMO Segregation and Identity Preservation.” Food Policy 27:81-99.

Elbehri, A. 2005. "Biopharming and the Food System: Examining the Potential Benefits and Risks." AgBioForum 8(1):18-25.

European Union. 2003. “GMOs: Commission Publishes Recommendations to Ensure CoExistence of GM and Non-GM Crops.” Press release IP/03/1096, 23 July.

—. 2004. "Questions and Answers on the Regulation of GMOs in the EU." MEMO/04/85, Brussels, 15 April.

Evenson, R.E., and D. Gollin. 2003. “Assessing the Impact of the Green Revolution, 1960 to 2000." Science 300(5620):758-762.

Falck-Zepeda, J.B., G. Traxler, and R.G. Nelson. 2000. "Surplus Distribution from the Introduction of a Biotechnology Innovation." American Journal of Agricultural Economics 82(2): 360-69.

Fischer, R., E. Stoger, S. Schillberg, P. Christou, and R.M. Twyman. 2004. "Plant-Based Production of Biopharmaceuticals." Current Opinion in Plant Biology 7:152-158.

Fulton, M., and K. Giannakas. 2004. "Inserting GM Products into the Food Chain: The Market and Welfare Effects of Different Labeling and Regulatory Regimes." American Journal of Agricultural Economics 86(1):42-60.

Goldstein, D.A., and J.A. Thomas. 2004. "Biopharmaceuticals Derived from Genetically Modified Plants.” QJM: An International Journal of Medicine 97:705-716.

Graff, G., and G. Moschini. 2004. "Pharmaceuticals and Industrial Products in Crops: Economic Prospects and Impacts on Agriculture.” Iowa Ag Review, Vol. 10, No. 4, Fall 2004.

Howard, J.A. 2005. "Commercialization of Biopharmaceutical and Bioindustrial Proteins from Plants.” Crop Science 45:468-472.

James, C. 2005. “Global Status of Biotech/GM Crops in 2005.” ISAAA Brief No. 34-2005, Ithaca, NY.

Kershen, D.L. 2004. "Legal Liability Issues in Agricultural Biotechnology." Crop Science 44: 456-463. 
Lapan, H., and G. Moschini. 2004. "Innovation and Trade with Endogenous Market Failure: The Case of Genetically Modified Products." American Journal of Agricultural Economics 86(3):630-644.

Ma, J-C., E. Barros, R. Bock, P. Christou, P.J. Dale, P.J. Dix, R. Fischer, J. Irwin, R. Mahoney, M. Pezzotti, S. Schillberg, P. Sparrow, E. Stoger, and R.M. Twyman. 2005. “Molecular Farming for New Drugs and Vaccines." EMBO Reports 6(7):593-599.

Ma J-C., P.M.W. Drake, and P. Christou. 2003. "The Production of Recombinant Pharmaceutical Proteins in Plants." Nature Reviews Genetics 4:794-805.

Miller, H.I., and D. Longtin. 2003. "Down on the Biopharm." Policy Review December 2003 and January 2004(122).

Moschini, G., H. Bulut, and L. Cembalo. 2005. "On the Segregation of Genetically Modified, Conventional and Organic Products in European Agriculture: A Multi-market Equilibrium Analysis." Journal of Agricultural Economics, 56(3):347-372.

Moschini, G., H. Lapan, and A. Sobolevsky. 2000. "Roundup Ready Soybeans and Welfare Effects in the Soybean Complex." Agribusiness 16(1):33-55.

NRC (National Research Council). 2004. Biological Confinent of Genetically Engineered Organisms, Washington, DC: The National Academies Press.

Peterson, R., and C. Arntzen. 2004. "On Risk and Plant-based Biopharmaceuticals." Trends in Biotechnology 22(2, February):64-66.

Pew Initiative. 2004. Issues in the regulation of genetically engineered plants and animals, Washington, D.C., Pew Initiative on Food and Biotechnology, April.

Scheller, J., and U. Conrad. 2005. "Plant-Based Material, Protein and Biodegradable Plastic." Current Opinion in Plant Biology 8:188-196.

Smyth, S., P.W.B. Phillips, W.A. Kerr, and G.G. Khachatourians. 2004. Regulating the Liabilities of Agricultural Biotechnology. New York: CABI Publishing.

Sobolevsky, A., G. Moschini, and H. Lapan. 2005. "Genetically Modified Crops and Product Differentiation: Trade and Welfare Effects in the Soybean Complex." American Journal of Agricultural Economics 87(3):621-644.

Taylor, M.R., and J.S. Tick. 2003. Post-Market Oversight of Biotech Foods: Is the System Prepared? Resources for the Future, Washington, D.C.

UCS (Union of Concerned Scientists). 2004. A Growing Concern: Protecting the Food Supply in an Era of Pharmaceutical and Industrial Crops. December. 
USDA/APHIS/BRS. 2006. Draft Guidance for APHIS Permits for Field Testing or Movement of Organisms with Pharmaceutical or Industrial Intent. March 31.

Wisner, R. 2005. The Economics of Pharmaceutical Crops-Potential Benefits and Risks for Farmers and Rural Communities. Report for the Union of Concerned Scientists. December. 